\title{
Cross-Coupling Chemistry at Mononuclear and Dinuclear Nickel Complexes
}

\author{
Sibo Lin, Theodor Agapie* \\ Division of Chemistry and Chemical Engineering, California Institute of Technology, MC127-72, 1200 E. California Blvd., \\ Pasadena, CA 91125, USA \\ Fax +1(626)3956948; E-mail: agapie@ caltech.edu \\ Received 12 October 2010
}

\begin{abstract}
Nickel catalysts have been developed for a wide range of $\mathrm{C}-\mathrm{C}$ bond formation reactions. Reaction mechanisms proposed for these transformations generally involve catalytic intermediates based on mononickel species. These reactions are reviewed and the possibility of dinickel species active in $\mathrm{C}-\mathrm{C}$ cross-coupling chemistry is discussed.
\end{abstract}

Key words: nickel, cross-coupling, alkyl halides, dinuclear mechanism, metal-arene interactions

Cross-coupling reactions have broad utility in organic synthesis. ${ }^{1}$ Recent catalyst developments have extended the versatility of these transformations to alkyl halides as cross-coupling partners. ${ }^{2}$ Reactions of species that possess $\beta$-hydrogen atoms have been challenging because of $\beta-\mathrm{H}$ elimination side reactions that lead to the formation of olefins. Following early reports of nickel-, iron-, copper-, and silver-mediated cross-coupling of unactivated alkyl halides displaying $\beta$-hydrogen atoms, ${ }^{3}$ a variety of catalysts have been developed based on diverse ligand sets and metals. ${ }^{2}$ The most versatile catalysts for coupling of alkyl electrophiles are based on nickel. ${ }^{2}$ An array of mechanisms have been proposed invoking metal oxidation states ranging from $\mathrm{Ni}^{0}$ through $\mathrm{Ni}^{\mathrm{IV}}$, but questions related to reaction mechanism and the effects various ligands have on catalyst behavior remain open. ${ }^{2}$ A more detailed understanding of these aspects is expected to lead to the development of more active and selective catalysts. Herein, we discuss the mechanisms typically proposed for cross-coupling chemistry with specific analyses of several nickel systems and highlight a recent report supporting a mechanism invoking dinickel intermediates.

A typical catalytic cycle proposed for cross-coupling chemistry (Scheme 1, mechanism A, left) involves formal two-electron processes. An alkyl halide undergoes oxidative addition at a low-oxidation-state metal complex (A) to generate a metal-alkyl halide $(\mathbf{B})$. Transmetallation leads to a metal-dialkyl species $(\mathbf{C})$ that undergoes reductive elimination to generate the organic product with a new $\mathrm{C}-\mathrm{C}$ bond and regenerating $\mathbf{A}$. This mechanism requires metal oxidation-state changes by two units between $\mathbf{M}^{\mathrm{m}}$ and $\mathbf{M}^{\mathrm{m}+2}$. Alternative mechanistic pathways involve one-electron processes. One variation (Scheme 1, mechanism $\mathrm{B}$, right) involves only two metal oxidation states,

SYNLETT 2011, No. 1, pp 0001-0005

Advanced online publication: 16.12.2010

DOI: 10.1055/s-0030-1259093; Art ID: P00110ST

(c) Georg Thieme Verlag Stuttgart · New York

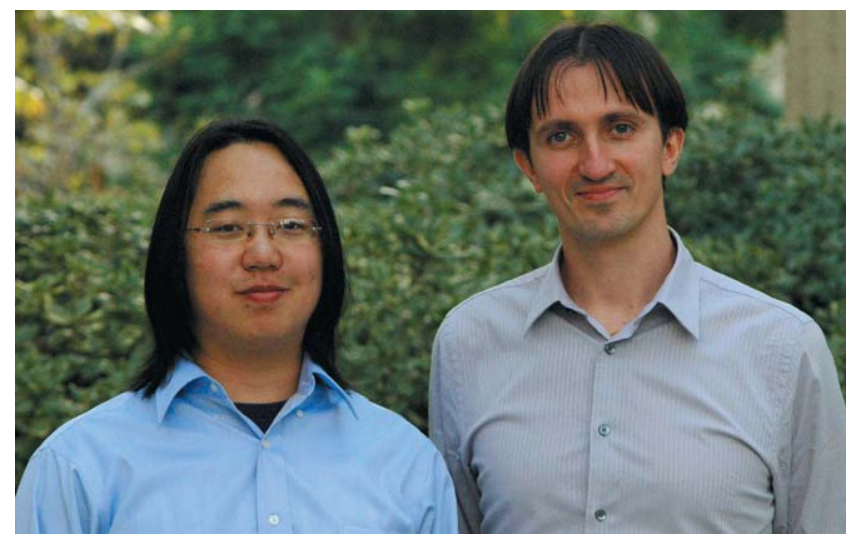

Sibo Lin (left) was raised in Evansville, Indiana. He conducted his undergraduate studies at the Indiana University and received his B.S. degrees in Chemistry and Mathematics in 2008. He is currently doing his Ph.D. study in Chemistry at the California Institute of Technology.

Theodor Agapie (right) was born in Bucharest, Romania. He received his B.S. degree from the Massachusetts Institute of Technology in 2001 and his Ph.D. from the California Institute of Technology in 2007. Upon completion of postdoctoral studies at the University of California, Berkeley, in 2009, he returned to the California Institute of Technology to start his independent career as an Assistant Professor of Chemistry. His current research is focused on the design and study of multinuclear metal complexes with applications in catalysis.

$\mathrm{M}^{\mathrm{m}} / \mathrm{M}^{\mathrm{m}+1}$. Metal-halide $\mathbf{D}$ undergoes transmetallation to form metal-alkyl E. Halogen atom abstraction from the alkyl halide to generate an alkyl radical $\left(\mathrm{R}^{1} \bullet\right)$ and metalalkyl halide $\mathbf{F}$. The intermediate radical $\left(\mathrm{R}^{1} \bullet\right)$ is proposed to lead to $\mathrm{C}-\mathrm{C}$ bond formation without binding to or oxidizing the metal center. ${ }^{4}$ Thus, high oxidation-state intermediates are formally avoided. Another mechanistic possibility (Scheme 1, mechanism C, right) involves species in three oxidation states, $\mathrm{M}^{\mathrm{m}} / \mathrm{M}^{\mathrm{m}+1} / \mathrm{M}^{\mathrm{m}+2}$, formally allowing for oxidative addition in two one-electron steps. Reductive elimination occurs from the higher oxidation state metal-dialkyl halide species G. The nickel-based mechanisms discussed below relate to mechanisms A-C in terms of the radical or non-radical nature of intermediates and the extent of variation of metal oxidation state, but the order of substrate reaction may vary.

Knochel established the feasibility of nickel-catalyzed cross-coupling of alkyl halides with minimal $\beta$-H elimination products. When a $\pi$-system (either tethered to the alkyl halide or as a separate additive) was present, coupling of alkyl bromides and iodides with dialkyl zinc reagents was observed. ${ }^{5}$ The catalytic cycle was proposed to 


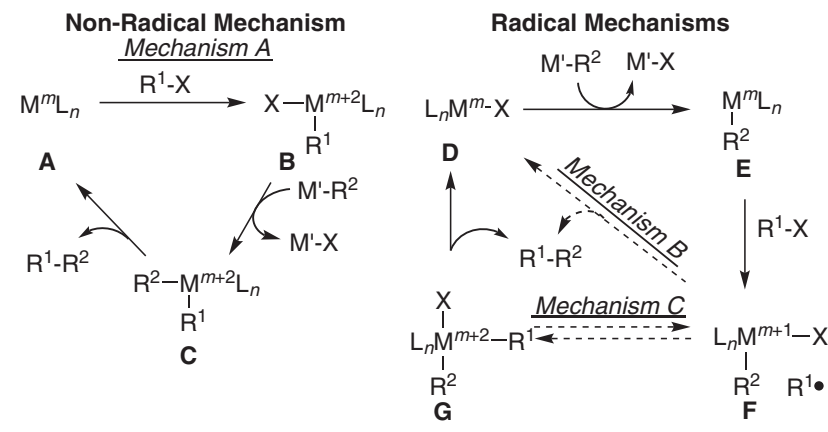

Scheme 1 Proposed mechanisms for cross-coupling catalysis

follow a $\mathrm{Ni}^{0} / \mathrm{Ni}^{\mathrm{II}}$ mechanism (Scheme 1 , mechanism $\mathrm{A}$ ) with the unsaturated moiety acting as a labile $\pi$-acid to $\mathrm{Ni}^{\mathrm{II}}$-alkyl halide intermediates and promoting reductive elimination to alkyl-alkyl products and $\mathrm{Ni}^{0}$ species (Scheme 2). Nickel-arene interactions have also been proposed for systems based on $\mathrm{Ni} / \mathrm{C}$ for the coupling of aryl Grignard reagents and aryl halides. ${ }^{6}$

Kambe and co-workers reported a butadiene-based catalytic system for coupling of alkyl halides and Grignard reagents. ${ }^{7} \mathrm{Ni}^{0}$ precursors react with two butadiene molecules to generate a $\mathrm{Ni}^{\mathrm{II}}$-diallyl species followed by nucleophilic attack by a Grignard reagent to yield a $\eta^{1}, \eta^{3}$ octadiene-diylnickelate complex (Scheme 3). Crosscoupling is proposed to occur by oxidative addition of an alkyl halide to generate $\mathrm{Ni}^{\mathrm{IV}}$-dialkyl species followed by reductive elimination to release the organic product and regenerate $\mathrm{Ni}^{\mathrm{II}}$-diallyl. It is noteworthy that the steps involved - transmetallation, oxidative addition, and reductive elimination - are in inverted order from the classical catalytic cycle (Scheme 1, mechanism A). The proposed $\mathrm{Ni} /{ }^{\mathrm{II}} \mathrm{Ni}^{\mathrm{IV}}$ cycle is supported by the reaction of $\mathrm{NiBr}_{2}$ with two equivalents of $n$-octylmagnesium chloride in the presence of isoprene. Octane and 1-octene were detected (43 and $45 \%$ yields, respectively), whereas the homocoupled product $n$-hexadecane was not. This indicates that $\mathrm{Ni}^{\mathrm{II}}$ alkyl halide species do not lead to productive crosscoupling, and thus that oxidative addition of alkyl halide by $\mathrm{Ni}^{0}$ is not operative. The non-radical nature of this mechanism is supported by the cross-coupling of a radical clock, (bromomethyl)cyclopropane, without observation of olefinic products.

$\mathrm{Ni}^{\mathrm{I}}$-methyl-terpyridine was found by Vicic and co-workers to catalyze Negishi coupling of alkyl bromides and iodides. ${ }^{8}$ Radical intermediates are supported by observations of minor homocoupled products from alkyl iodide substrates and substantial formation of olefinic products upon reaction with the radical clock (iodomethyl)cyclopropane. Based on stoichiometric reactivity studies, isolated intermediates and computational studies, a $\mathrm{Ni}^{\mathrm{I}} / \mathrm{Ni}^{\mathrm{III}}$ catalytic cycle is proposed by the authors (Scheme 4). Although similar to mechanism $\mathrm{C}$, generation of the alkyl radical by halogen atom abstraction is proposed to lead initially to terpyridine ligand oxidation rather than metal oxidation.

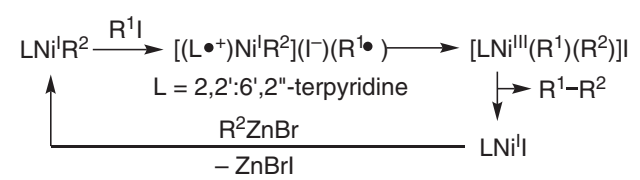

Scheme 4 Proposed mechanism for Ni-terpyridine catalyzed crosscoupling of alkyl halides and zinc alkyl reagents ${ }^{8 a}$

A variety of nickel-based catalysts that catalyze coupling reactions with alkyl electrophiles have been reported by $\mathrm{Fu}$ and co-workers. Bathophenanthroline-nickel systems (Figure 1a) are active for Suzuki and Hiyama coupling of alkyl bromides and iodides. ${ }^{9}$ Pybox-nickel catalysts (Figure 1b) promote Negishi cross-coupling of a variety of alkyl halides. ${ }^{10}$ Diamine-nickel systems (Figure 1c) perform Suzuki cross-coupling of alkyl bromides and chlorides. ${ }^{11}$ Amino alcohol-nickel systems are active for Suzuki and Hiyama couplings (Figure 1d). ${ }^{12}$ Bis(oxazoline)-nickel catalysts are active for Kumada coupling of $\alpha$-bromoketones. ${ }^{13}$ With the exception of bathophenanthroline, chiral versions of these ligands have also been developed; the corresponding cross-coupling of racemic mixtures of alkyl halides possessing chiral halogen-bearing carbon centers often yields a single enantiomer. No kinetic resolution is observed during these reactions, implicating an alkyl radical intermediate as being involved

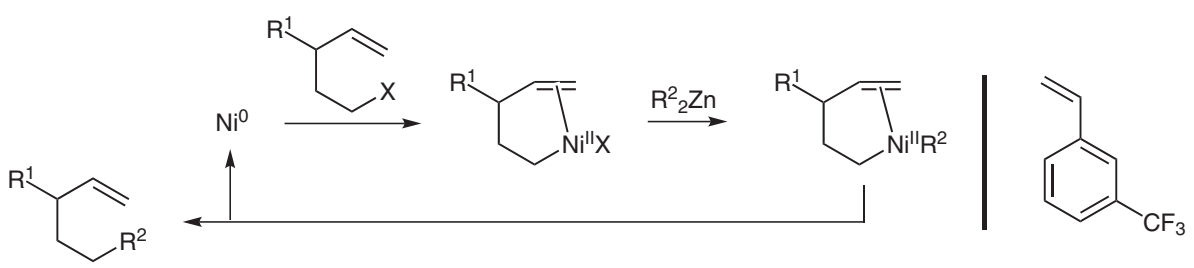

Scheme 2 Proposed mechanism for nickel-catalyzed cross-coupling of dialkyl zinc and unsaturated alkyl halides (left) and a representative additive for the cross-coupling of saturated alkyl halides (right) ${ }^{5}$

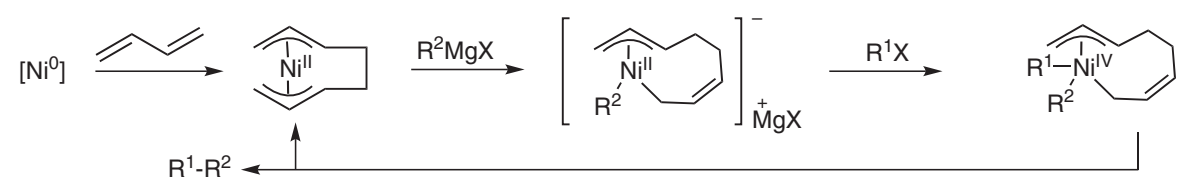

Scheme 3 Proposed mechanism for Ni-butadiene catalyzed cross-coupling of alkyl halides and Grignard reagents ${ }^{7}$ 
in the mechanism for stereoconvergence. While a detailed mechanism has not been proposed, the authors note that preliminary kinetics data for the bis(oxazoline) systems were consistent with a variant of mechanism $\mathrm{C}$, as proposed by Vicic and co-workers (Scheme 4). ${ }^{13}$

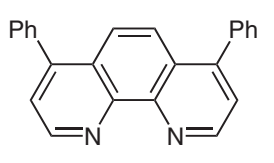

a

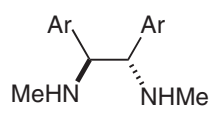

c

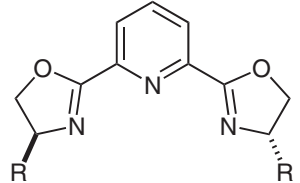

b

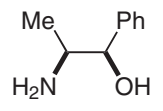

d
Figure 1 Examples of ligands utilized for nickel-catalyzed crosscouplings ${ }^{9-12}$

Nickel catalysts supported by terpyridine, pybox, or amino alcohol ligands were further studied by Cárdenas and $\mathrm{Fu}$ for cascade cyclization and cross-coupling of alkyl zinc halides with iodo-olefins bearing pendant olefins. ${ }^{12 b, 14}$ The mechanism of this reaction was proposed to be reminiscent of that shown in Scheme 4, with the intermediate alkyl radical $\left(\mathrm{R}^{1} \bullet\right.$ ) performing intramolecular cyclization prior to coordination to nickel. The presence of substrate-based radical intermediates was supported by experiments with radical clocks. Because involvement of ancillary ligands in redox chemistry is dependent on their electronic structure, ligands that are harder to oxidize may follow mechanism $\mathrm{C}$ (or $\mathrm{B}$ ), with oxidation of the metal center rather than of the ligand.

A nickel complex supported by an amidobis(amine) tridentate pincer ligand $\left({ }^{\mathrm{Me}} \mathrm{NN}_{2}\right.$ ) was reported by $\mathrm{Hu}$ to catalyze Sonogashira coupling of unactivated alkyl halides and Kumada coupling of unactivated alkyl iodides and bromides. ${ }^{15}$ Kumada couplings with $\mathrm{CH}_{2} \mathrm{Cl}_{2}$ and $\mathrm{CHCl}_{3}$ provide rare examples of efficient di- and tricoupled products, respectively. ${ }^{15 \mathrm{~b}} \mathrm{~A}$ radical mechanism for Kumada coupling is suggested by (a) faster reactivity of $\mathrm{CH}_{2} \mathrm{Cl}_{2}$ and $\mathrm{CHCl}_{3}$ versus octyl chloride; (b) retarded reaction in the presence of radical trap TEMPO, and (c) olefinic products from coupling with the radical clock (bromomethyl)cyclopropane. For alkyl Grignard reagents, a $\mathrm{Ni}^{\mathrm{II}} / \mathrm{Ni}^{\mathrm{IV}}$ catalytic cycle similar to mechanism $\mathrm{C}$ is proposed by the authors (Scheme 5, top). A different mechanism appears to be active for aryl Grignard reagents. The lack of crosscoupling chemistry between $\left({ }^{\mathrm{Me}} \mathrm{NN}_{2}\right) \mathrm{NiPh}$, an active precatalyst, and octyl iodide or $\mathrm{PhMgCl}$ was interpreted in terms of a $\mathrm{Ni}^{\mathrm{II}} / \mathrm{Ni}^{\mathrm{IV}}$ catalytic cycle with the formation of a Ni-alkyl-diaryl intermediate prior to reductive elimination (Scheme 5, bottom). Oxidation of the ancillary ligand to form a $\mathrm{Ni}^{\mathrm{III}}$ complex was presented as a possible alternative to avoid $\mathrm{Ni}^{\mathrm{IV}}$ intermediates. The nature of the intermediate nickel species generated upon halogen atom abstraction from alkyl halides also remains unclear.

As discussed above, mechanistic proposals for crosscoupling reactions commonly assume a mononuclear catalytic cycle. However, for similarly stereotyped palladiumcatalyzed cross-couplings, recent work by Ritter and Sanford suggest that dinuclear species can be active for C-heteroatom coupling chemistry. ${ }^{16}$ Our group has recently reported $\mathrm{C}-\mathrm{C}$ bond formation from dinuclear $\mathrm{Ni}$ complexes. ${ }^{17}$

Dinuclear nickel complexes supported by a terphenyl diphosphine (1-3; Scheme 6) were synthesized and shown to present interactions between the $\pi$-system of the central arene and the metal centers. ${ }^{17}$ These interactions

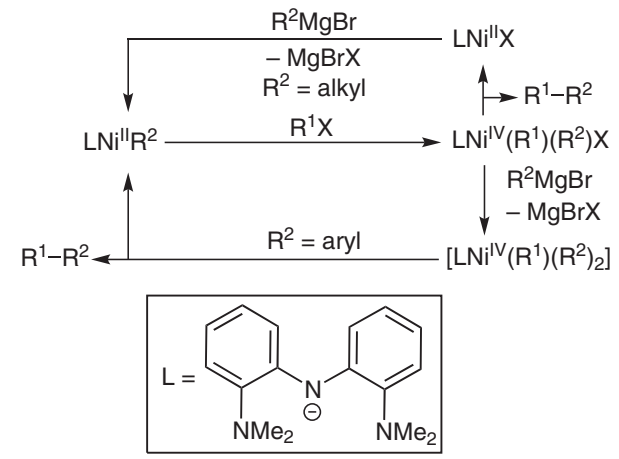

Scheme 5 Proposed mechanisms for Ni-amidobis(amine) catalyzed cross-coupling of alkyl halides and Grignard reagents ${ }^{15 c-e}$

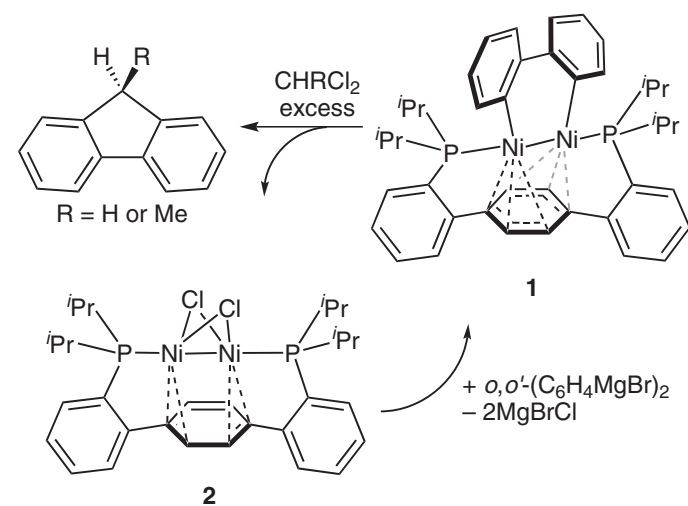

Scheme 6 Reactivity of $\mathrm{Ni}_{2}{ }_{2}$-biphenyldiyl (1) with gem-dichloroalkanes and $\mathrm{CO}^{17}$ 
are reminiscent of those proposed by Knochel to be crucial for nickel catalysts based on additives such as olefins and electron-deficient arenes. ${ }^{5}$ Reaction of $\mathrm{Ni}_{2}{ }_{2}$-biphenyldiyl complex 1 (Scheme 6) with gem-dichloroalkanes $\mathrm{CHRCl}_{2}(\mathrm{R}=\mathrm{Me}, \mathrm{H})$ affords fluorenes and $\mathrm{Ni}_{2}{ }_{2}$-dichloride complex 2 . These transformations involve a rare example of the formation of two $\mathrm{C}-\mathrm{C}$ bonds. Stoichiometric intramolecular $\mathrm{C}-\mathrm{C}$ couplings have been reported for $\mathrm{Ni}_{2}{ }_{2}$ dinuclear complexes $;{ }^{18}$ in those cases the metal centers are reduced to $\mathrm{Ni}^{0}$. The $\mathrm{Ni}_{2}{ }_{2}$ core in the present case suggests an intermolecular dinuclear mechanism for $\mathrm{C}-\mathrm{C}$ bond formation (Scheme 7). Oxidative addition of a $\mathrm{C}-\mathrm{Cl}$ bond yields $\mathrm{Ni}_{2}{ }_{2}$ intermediate $\mathbf{H}$ (Scheme 7 , top). Reductive elimination of chloromethyl and aryl groups generates a $\mathrm{Ni}_{2}{ }_{2}$-arylchloride $\mathbf{I}$, which undergoes intramolecular oxidative addition of the pendant $\mathrm{C}-\mathrm{Cl}$ bond to yield the $\mathrm{Ni}_{2}{ }_{2}$ species J. Reductive elimination of the remaining organic ligands yields fluorene. Thus, this cross-coupling reaction is proposed to utilize a $\mathrm{Ni}_{2}{ }_{2} / \mathrm{Ni}^{\mathrm{II}}{ }_{2}$ cycle instead of a $\mathrm{Ni}^{\mathrm{n}} /$ $\mathrm{Ni}^{\mathrm{n}+2}$ mechanism. The oxidation state of each nickel center of the dinuclear unit changes by only one unit, in contrast to the mononuclear mechanisms invoking two-unit oxidation state changes for concerted oxidative addition or reductive elimination steps. A mixed-valence $\mathrm{Ni}_{2}^{\mathrm{I}} / \mathrm{Ni}^{\mathrm{I}}$ $\mathrm{Ni}^{\mathrm{II}}$ cycle involving alkyl radical intermediates is also possible (Scheme 7, bottom). In that case, the high oxidation state intermediates $\left(\mathrm{Ni}^{\mathrm{I}} \mathrm{Ni}^{\mathrm{II}}\right)$ can preserve a partial $\mathrm{Ni}-$ $\mathrm{Ni}$ bond. The reaction between 1 and gem-dichloroethane to generate 9-methylfluorene is notable because it extends double $\mathrm{C}-\mathrm{C}$ coupling chemistry beyond $\mathrm{C}_{1}$ fragments, unlike the chemistry reported by $\mathrm{Hu}$. Intermediates derived from gem-dichloroethane display $\beta$-hydrogen atoms but still lead to the formation of 9-methylfluorene, indicating that $\mathrm{C}-\mathrm{C}$ bond formation is faster than processes derived from $\beta$-H elimination.

As another route to $\mathrm{C}-\mathrm{C}$ coupling, $\mathbf{1}$ was treated with $\mathrm{CO}$ to yield fluorenone and $\mathrm{Ni}^{0}{ }_{2}$-carbonyl complex $\mathbf{3}$ (Scheme 6). This is proposed to occur by coordination of $\mathrm{CO}$ and insertion into a metal-aryl bond, followed by the formation of a second $\mathrm{C}-\mathrm{C}$ bond by reductive elimination. Whereas the formation of fluorenone from mononuclear and dinuclear nickel complexes has been observed previously, this reaction is noteworthy because it generates a well-defined $\mathrm{Ni}_{2}{ }_{2}$ complex $(\mathbf{3}){ }^{18,19}$ The reactivity of $\mathbf{1}$ with $\mathrm{CHRCl}_{2}$ and $\mathrm{CO}$ suggests that the terphenyl diphosphine is capable of supporting $\mathrm{Ni}_{2}$ oxidation states ranging from $\mathrm{Ni}_{2}{ }_{2}$ to $\mathrm{Ni}^{\mathrm{I}} \mathrm{Ni}^{\mathrm{II}}$ or $\mathrm{Ni}^{\mathrm{II}}{ }_{2}$, indicating the potential for three- or four-electron redox transformations.

In summary, many catalysts based on nickel and other metals have been shown to mediate cross-coupling chemistry and several studies have provided mechanistic insights. Details regarding the nature of the active species and the oxidation state of the metal center, however, still remain unclear. The reactions described in this highlighted paper suggest dinuclear nickel complexes are an alternative to mononuclear species for catalytic intermediates in cross-coupling chemistry. The possibility for some of

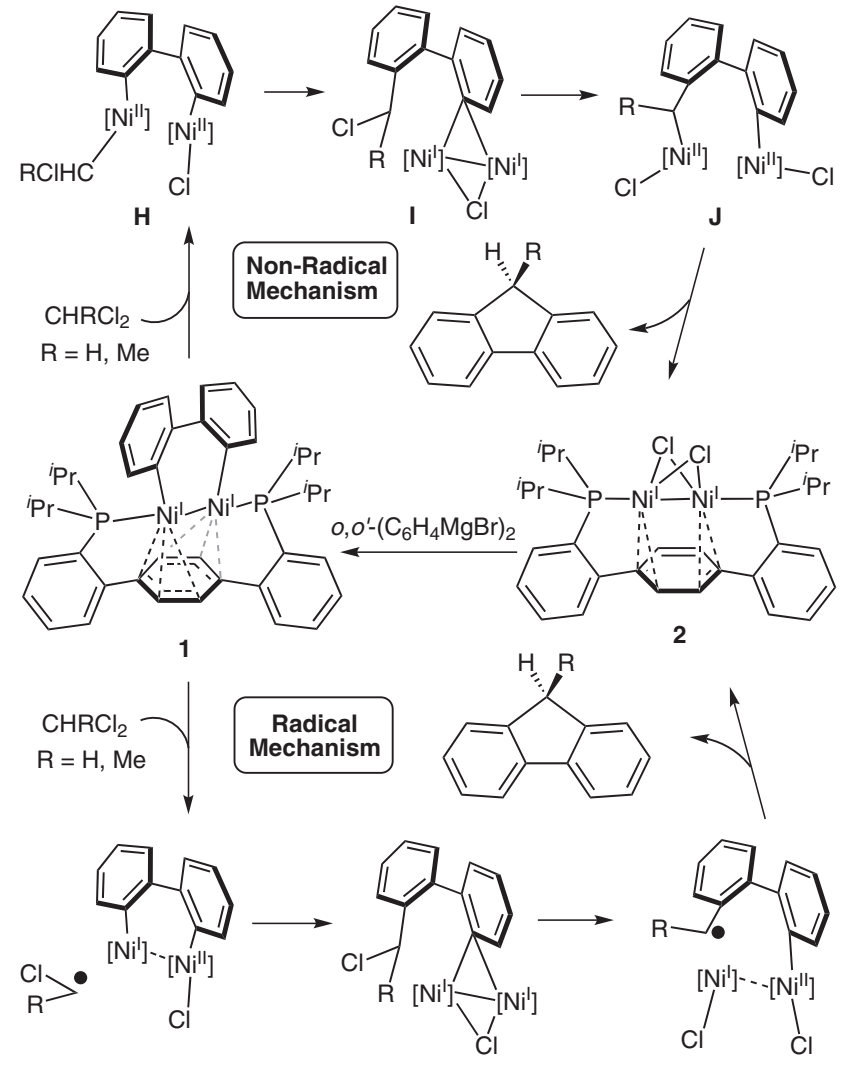

Scheme 7 Proposed mechanisms for formation of fluorene at a dinickel core $^{17}$

the previously reported systems (especially those without multidentate supporting ligands) to involve bimetallic catalysts is intriguing. Current work is focused on exploring dinuclear nickel cores with an emphasis on developing new multielectron transformations, detailing their reaction mechanisms, and understanding the role of the ligand framework in modulating organometallic reactivity.

\section{Acknowledgment}

We are grateful to BP, Caltech, and NSF GRFP (S.L.) for financial support.

\section{References and Notes}

(1) (a) Metal-Catalyzed Cross-Coupling Reactions, 2nd ed.; de Meijere, A.; Diederich, F., Eds.; Wiley-VCH: Weinheim, 2004. (b) Transition Metals for Organic Synthesis, 2nd ed.; Beller, M.; Bolm, C., Eds.; Wiley-VCH: Weinheim, 2004.

(2) (a) Terao, J.; Kambe, N. Acc. Chem. Res. 2008, 41, 1545. (b) Netherton, M.; Fu, G. Adv. Synth. Catal. 2004, 346, 1525. (c) Rudolph, A.; Lautens, M. Angew. Chem. Int. Ed. 2009, 48, 2656. (d) Frisch, A. C.; Beller, M. Angew. Chem. Int. Ed. 2005, 44, 674.

(3) (a) Kochi, J. K.; Tamura, M. J. Am. Chem. Soc. 1971, 93, 1483. (b) Kochi, J. K.; Tamura, M. J. Am. Chem. Soc. 1971, 93, 1485. (c) Tamao, K.; Hiyama, T.; Niegishi, E.-i.

J. Organomet. Chem. 2002, 653, 1. (d) Tamura, M.; Kochi, J. K. J. Am. Chem. Soc. 1971, 93, 1487. (e) Uchino, M.; Yamamoto, A.; Ikeda, S. J. Organomet. Chem. 1970, 24, C63. (f) Yamamoto, A. J. Organomet. Chem. 2002, 653, 5. 
(4) (a) Organometallic Mechanisms and Catalysis, 1st ed.; Kochi, J. K., Ed.; Academic Press, Inc.: New York, 1978. (b) Hatakeyama, T.; Hashimoto, T.; Kondo, Y.; Fujiwara, Y.; Seike, H.; Takaya, H.; Tamada, Y.; Ono, T.; Nakamura, M. J. Am. Chem. Soc. 2010, 132, 10674.

(5) (a) Devasagayaraj, A.; Stüdemann, T.; Knochel, P. Angew. Chem. Int. Ed. Engl. 1996, 34, 2723. (b) Giovannini, R.; Knochel, P. J. Am. Chem. Soc. 1998, 120, 11186.

(c) Giovannini, R.; Studemann, T.; Devasagayaraj, A.; Dussin, G.; Knochel, P. J. Org. Chem. 1999, 64, 3544. (d) Giovannini, R.; Stüdemann, T.; Dussin, G.; Knochel, P. Angew. Chem. Int. Ed. 1998, 37, 2387.

(6) Tasler, S.; Lipshutz, B. H. J. Org. Chem. 2002, 68, 1190.

(7) Terao, J.; Watanabe, H.; Ikumi, A.; Kuniyasu, H.; Kambe, N. J. Am. Chem. Soc. 2002, 124, 4222.

(8) (a) Jones, G. D.; Martin, J. L.; McFarland, C.; Allen, O. R.; Hall, R. E.; Haley, A. D.; Brandon, R. J.; Konovalova, T.; Desrochers, P. J.; Pulay, P.; Vicic, D. A. J. Am. Chem. Soc. 2006, 128, 13175. (b) Anderson, T. J.; Jones, G. D.; Vicic, D. A. J. Am. Chem. Soc. 2004, 126, 8100.

(9) (a) Lee, J.-Y.; Fu, G. C. J. Am. Chem. Soc. 2003, 125, 5616. (b) Powell, D. A.; Fu, G. C. J. Am. Chem. Soc. 2004, 126, 7788.

(10) (a) Arp, F. O.; Fu, G. C. J. Am. Chem. Soc. 2005, 127, 10482. (b) Fischer, C.; Fu, G. C. J. Am. Chem. Soc. 2005, 127, 4594. (c) Smith, S.; Fu, G. Angew. Chem. Int. Ed. 2008, 47, 9334. (d) Son, S.; Fu, G. C. J. Am. Chem. Soc. 2008, 130, 2756.
(11) (a) Saito, B.; Fu, G. C. J. Am. Chem. Soc. 2007, 129, 9602. (b) Saito, B.; Fu, G. C. J. Am. Chem. Soc. 2008, 130, 6694. (c) Owston, N. A.; Fu, G. C. J. Am. Chem. Soc. 2010, 132, 11908.

(12) (a) Dai, X.; Strotman, N. A.; Fu, G. C. J. Am. Chem. Soc. 2008, 130, 3302. (b) González-Bobes, F.; Fu, G. C. J. Am. Chem. Soc. 2006, 128, 5360. (c) Strotman, N.; Sommer, S.; Fu, G. Angew. Chem. Int. Ed. 2007, 46, 3556.

(13) Lou, S.; Fu, G. C. J. Am. Chem. Soc. 2010, 132, 1264.

(14) Phapale, V.; Buñuel, E.; García-Iglesias, M.; Cárdenas, D. Angew. Chem. Int. Ed. 2007, 46, 8790.

(15) (a) Vechorkin, O.; Barmaz, D.; Proust, V.; Hu, X. J. Am. Chem. Soc. 2009, 131, 12078. (b) Csok, Z.; Vechorkin, O.; Harkins, S. B.; Scopelliti, R.; Hu, X. J. Am. Chem. Soc. 2008, 130, 8156. (c) Vechorkin, O.; Csok, Z.; Scopelliti, R.; Hu, X. Chem. Eur. J. 2009, 15, 3889. (d) Vechorkin, O.; Hu, X. Angew. Chem. Int. Ed. 2009, 48, 2937. (e) Vechorkin, O.; Proust, V.; Hu, X. J. Am. Chem. Soc. 2009, 131, 9756.

(16) (a) Deprez, N. R.; Sanford, M. S. J. Am. Chem. Soc. 2009, 131, 11234. (b) Powers, D. C.; Ritter, T. Nat Chem. 2009, 1, 302. (c) Powers, D. C.; Xiao, D. Y.; Geibel, M. A. L.; Ritter, T. J. Am. Chem. Soc. 2010, 132, 14530.

(17) Velian, A.; Lin, S.; Miller, A. J. M.; Day, M. W.; Agapie, T. J. Am. Chem. Soc. 2010, 132, 6296.

(18) (a) Ramakrishna, T. V. V.; Sharp, P. R. Organometallics 2004, 23, 3079. (b) Eisch, J. J.; Piotrowski, A. M.; Han, K. I.; Kruger, C.; Tsay, Y. H. Organometallics 1985, 4, 224.

(19) Edelbach, B. L.; Lachicotte, R. J.; Jones, W. D. Organometallics 1999, 18, 4040. 